\title{
Microinjection of Superior Cervical Ganglion Neurons for Studying Axon Degeneration
}

\author{
Jonathan Gilley and Andrea Loreto
}

\begin{abstract}
Primary cultures of neurons of the peripheral nervous system have been successfully used for studying many aspects of neuronal development and survival, including investigations into the mechanisms of axon degeneration. In this chapter, we describe how to prepare and microinject dissociated cultures of sympathetic neurons of the superior cervical ganglion (SCG) specifically for use in highly controlled and targeted assays of axon survival and degeneration.
\end{abstract}

Key words Superior cervical ganglion, Dissociated SCG culture, Microinjection, Axon survival, Axon degeneration, Axon transection, Wallerian degeneration

\section{Introduction}

Primary cultures of early postnatal sympathetic neurons of the superior cervical ganglion (SCG), which are dependent on nerve growth factor (NGF) for survival, are an established model system for studying many aspects of neuronal biology. SCG neurons predominantly extend axon-like neurites [1], and these can grow several millimeters in just a few days making them ideally suited to investigations of axonal function. Indeed, studies in SCG cultures, together with studies in primary cultures of mid-to-late embryonic stage sensory neurons of the dorsal root ganglion (DRG), have contributed significantly to our understanding of the key molecular regulators of axon degeneration caused by physical injury (Wallerian degeneration) or related degeneration triggered by chemical insults $[2,3]$. Crucially, similar mechanisms appear to be involved during the degeneration of axons in a number of neurodegenerative disorders [2].

Targeted suppression of gene expression by RNA interference and exogenous expression of wild-type or modified proteins are useful tools for probing the roles of specific genes in cellular processes. SCG and DRG neuron cultures are relatively resistant to 
standard chemical transfection, so viral transduction of constructs has become a favored method for achieving these outcomes, especially in DRG explant or spot cultures (see Chapters 4 and 5). However, microinjection has also proved successful for delivery of expression vectors and other materials into cultured neurons and, in particular, SCG neurons, where larger cell bodies/nuclei and a greater ability to tolerate injection stresses make the process much more efficient than for DRG neurons or other neuron types (although DRG neurons can be injected, if desired). Microinjection is a technically challenging and low-throughput method targeting relatively small numbers of cells. While this makes it unsuited to biochemical analyses, it does provide a unique degree of precision that is particularly useful in studies of neurite (axon) degeneration in primary neuron cultures. For example, it provides a greater degree of control over the concentrations of the materials being introduced, as well as greater flexibility over the type and combinations of material that can be used. The ability to target small numbers of neurons in specific locations can also provide better spatial resolution of individual neurites than nontargeted methods. In addition, after an initial financial outlay on some specialized equipment, subsequent costs are relatively low and the ability to use standard mammalian expression vectors in injections, with no theoretical size restriction, is a particular advantage.

In this chapter, we present core methods for preparing and microinjecting primary cultures of dissociated mouse SCG neurons with the specific aim of studying neurite degeneration. A procedure for the dissociation of mouse SCG neurons, which is adapted from an earlier protocol for the dissociation of rat SCG neurons [4], is described first. Dissociated cultures are required, so that individual neurons can be targeted easily. Rat SCG neurons can be used if preferred, but most studies of neurite degeneration are performed in mouse cultures due to the growing number of useful mutant mouse models with altered axon survival $[2,3]$. Our method also describes a minor modification for the preparation of independent cultures from individual mice, for example, if litters of mixed genotypes are being processed. We next describe the basic procedure for microinjection of SCG neurons for the specific purpose of tracking the survival of their neurites. Crucially, in addition to any test materials being injected, a means for fluorescent labeling must be included in order that the injected neurons and their neurites can be visualized separately from the mass culture (Fig. 1). While we do not provide specific details, injected test materials have, in the past, included mammalian expression vectors and small interfering RNAs (siRNAs) [5-11], although purified proteins and other compounds can also be injected. We additionally describe a simple method for transecting the labeled neurites with a scalpel blade to trigger Wallerian degeneration, if required (Fig. 1). 
A
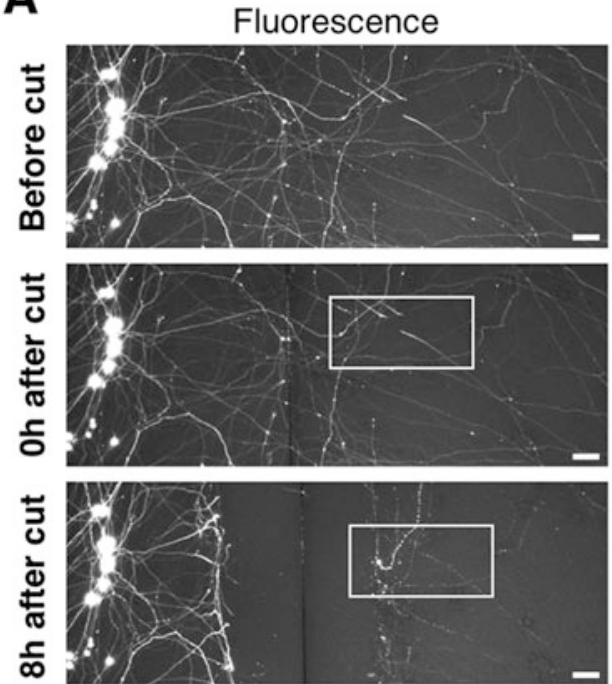

B

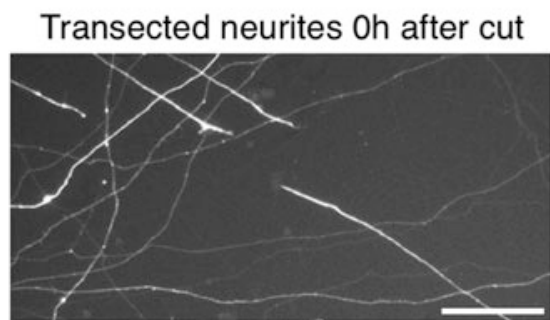

Phase-contrast
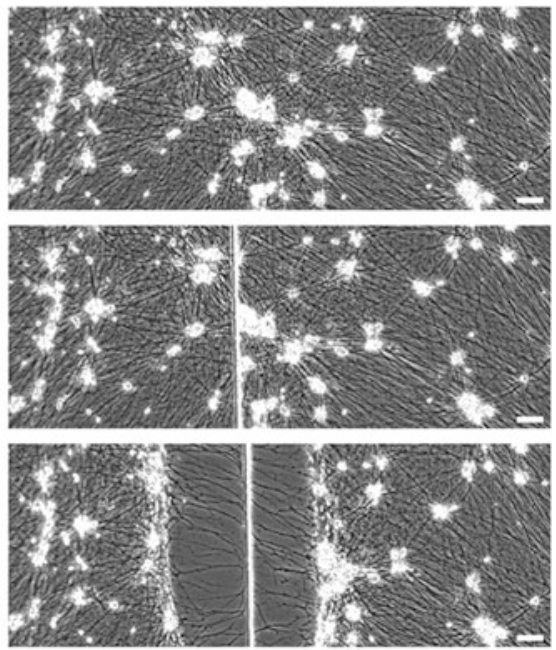

Transected neurites $8 \mathrm{~h}$ after cut

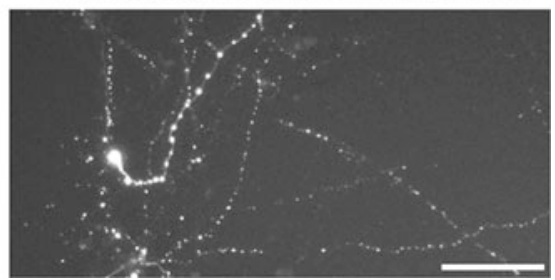

Fig. 1 Representative images of a generic microinjection experiment showing degeneration of labeled neurites after transection. (a) Low-magnification fluorescence and phase-contrast images of a region of a dissociated culture of wild-type SCG neurons $48 \mathrm{~h}$ after injection of a small cluster of the neurons with a fluorescent protein expression vector (pDsRed2). The series of images shows the same area before, immediately after $(0 \mathrm{~h})$, and $8 \mathrm{~h}$ after the introduction of a cut with a scalpel blade. Labeled cell bodies of the injected neurons are seen to the left of the fluorescence images with some of their labeled neurites extending to the right, the distal ends of which become disconnected from their cell bodies following transection and degenerate within $8 \mathrm{~h}$. Phase-contrast images reveal that changes in the relatively few labeled neurites are occurring within a dense network of unlabeled SCG neurons and neurites that appear grossly unaffected except for a small amount of retraction and neurite regeneration occurring at the cut site. (b) Higher magnification fluorescence images of the boxed regions in (a) for better visualization of the complete loss of continuity of the labeled, transected neurites by $8 \mathrm{~h}$ after cut. Scale bars $=100 \mu \mathrm{m}$

Finally, although this chapter is limited to a description of microinjection as a tool for assessing the survival of individual neurites (intact or transected) in response to various injected materials, it is important to note that there are many other potential applications of the technique. For example, microinjection of SCG neurons has already proven useful for live-imaging studies of axonal transport $[7,11,12]$ and could easily be applied to other aspects of axon biology, such as regeneration. 


\section{Materials}

\subsection{Dissociation and Culture of SCG Neurons}

Solution preparation and coating of dishes should be performed in a laminar flow cabinet or Class II biological safety cabinet. Sterile, ultrapure water (deionized, filtered water purified to $18.2 \mathrm{M} \Omega \cdot \mathrm{cm}$ resistivity at $25^{\circ} \mathrm{C}$ ) should be used for solutions, as applicable.

1. Trypsin solution: Dissolve lyophilized trypsin from bovine pancreas in $\operatorname{PBS}(-)$ (phosphate-buffered saline withoutcalcium chloride and magnesium chloride) at a concentration of $0.025 \%$. Alternatively, the $0.025 \%$ working solution can be diluted from a $0.25 \%$ concentrated stock solution. Store aliquots at $-20{ }^{\circ} \mathrm{C}$.

2. Collagenase solution: Dissolve desiccated type 2 collagenase (storage at $4{ }^{\circ} \mathrm{C}$ ) in PBS (withcalcium chloride and magnesium chloride) at a concentration of $0.2 \%$, and filter sterilize through a $0.22 \mu \mathrm{m}$ filter. Large batches can be prepared and aliquots stored at $-20{ }^{\circ} \mathrm{C}$; however, best results are obtained with freshly prepared solution. Activity of the desiccated collagenase may vary between batches, so incubation times should be adjusted accordingly.

3. SCG medium: DMEM (with $4500 \mathrm{mg} / 1$ glucose and $110 \mathrm{mg} / \mathrm{l}$ sodium pyruvate) supplemented with $10 \%$ fetal bovine serum (FBS) (see Note 1), $1 \times$ penicillin/streptomycin, $2 \mathrm{mM} \mathrm{L-glu-}$ tamine, $2-4 \mu \mathrm{g} / \mathrm{ml}$ aphidicolin (see Note 2 ), and $25-50 \mathrm{ng} / \mathrm{ml}$ 2.5S NGF or $100 \mathrm{ng} / \mathrm{ml} 7 \mathrm{~S} \mathrm{NGF} \mathrm{(see} \mathrm{Note} \mathrm{3).} \mathrm{Concentrated}$ stock solutions of each supplement are as follows: $100 \times$ penicillin/streptomycin $(10,000 \mathrm{U} / \mathrm{ml}$ penicillin and $10,000 \mu \mathrm{g} /$ $\mathrm{ml}$ streptomycin in $10 \mathrm{mM}$ citrate buffer), $100 \times$ L-glutamine $(200 \mathrm{mM}), 4 \mathrm{mg} / \mathrm{ml}$ aphidicolin in DMSO, and $100 \mu \mathrm{g} / \mathrm{ml}$ $2.5 \mathrm{~S}$ or $7 \mathrm{~S} \mathrm{NGF}$ prepared as per supplier instructions. Aliquots of each supplement are stored at $-20{ }^{\circ} \mathrm{C}$. SCG medium without aphidicolin and NGF can be stored for several weeks at $4{ }^{\circ} \mathrm{C}$ with the two omitted items being added fresh as needed.

4. Low-sided (uncoated) $35 \mathrm{~mm} \mu$-Dishes (ibidi) coated with poly-L-lysine and laminin (see Note 4): First, cover just the uncoated coverslip insert bases of the dishes with $1 \mathrm{ml}$ of a solution of $20 \mu \mathrm{g} / \mathrm{ml}$ poly-L-lysine hydrobromide $(\mathrm{Mw} \geq 300,000)$ dissolved in water. The working poly-L-lysine solution can be diluted from aliquots of a $2.5 \mathrm{mg} / \mathrm{ml}$ stock solution in water (store at $-20^{\circ} \mathrm{C}$ ). After at least $1 \mathrm{~h}$ at room temperature, remove the poly-L-lysine solution and wash the coverslip insert base once with sterile, ultrapure water before leaving to air dry completely. Dried poly-L-lysine-coated dishes can be stored for several weeks at $4{ }^{\circ} \mathrm{C}$ wrapped in parafilm. For subsequent laminin coating, $1 \mathrm{mg} / \mathrm{ml}$ laminin (stored as $20 \mu \mathrm{l}$ aliquots in $1.5 \mathrm{ml}$ microfuge tubes at $-20{ }^{\circ} \mathrm{C}$ ) is diluted to 
$20 \mu \mathrm{g} / \mathrm{ml}$ in DMEM $(980 \mu \mathrm{l}$ per $20 \mu \mathrm{l}$ aliquot $)$ and $\sim 50-100 \mu \mathrm{l}$ of the solution used to coat a small circular area, typically 7-10 $\mathrm{mm}$ in diameter, in the center of each coverslip insert base. It is important not to use SCG medium to dilute the laminin as the presence of FBS will interfere with coating. After l-2 $\mathrm{h}$ at room temperature, the laminin solution is aspirated immediately prior to addition of the dissociated neurons. The laminin-coated area should not be allowed to dry.

\subsection{Microinjection}

1. A complete microinjection system setup for injecting on an inverted microscope with a $32 \times / 40 \times$ objective and optional $4 \times / 5 \times$ objective and a holder for a $35 \mathrm{~mm}$ dish (see Note 5 ). The microscope should sit on an anti-vibration table. The procedures for injection described in this chapter are based on an Eppendorf system (Fig. 2). In it, an Eppendorf FemtoJet ${ }^{\circledR}$ microinjector pressurizes an injection capillary that is attached to a high resolution $\mathrm{x}, \mathrm{y}, \mathrm{z}$ motor module and an Eppendorf TransferMan $^{\circledR}$ micromanipulator. The micromanipulator allows proportional control over capillary movements. The

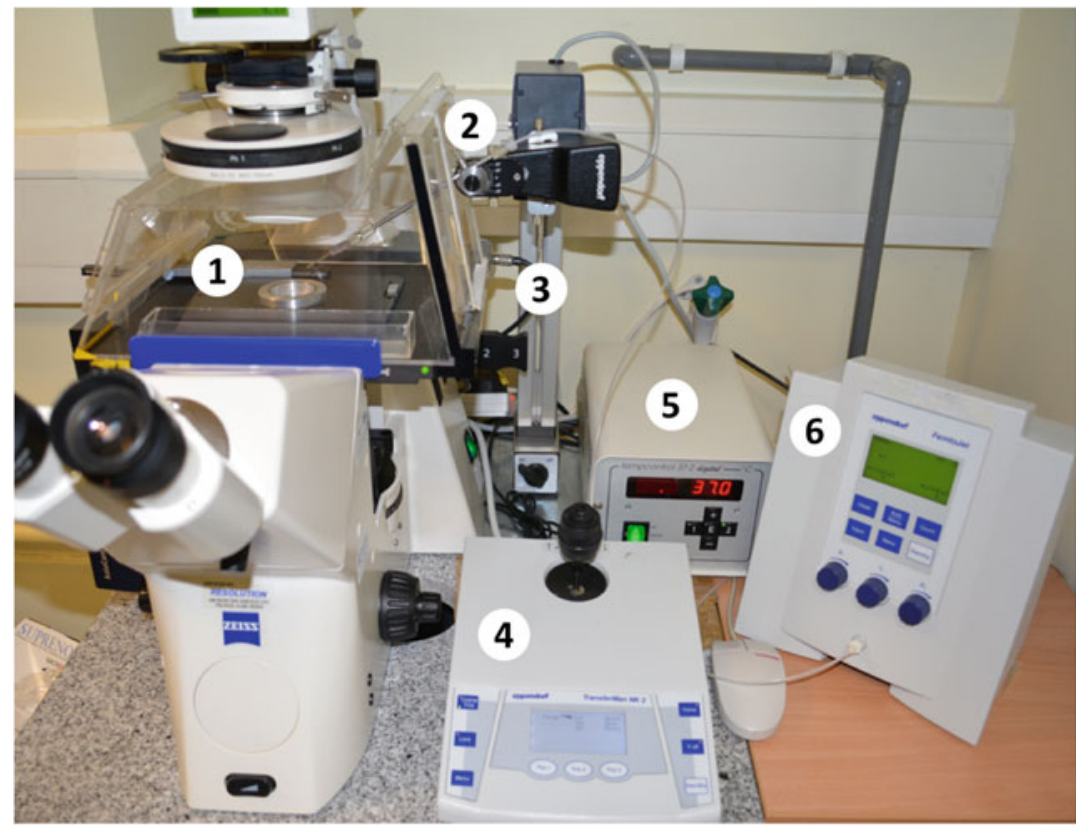

(1) Heated stage and environment chamber

(2) Motor module with capillary holder attached

3 Universal stand
(4) TransferMan ${ }^{\circledast}$ micromanipulator

(5) Temperature controller for heated stage

6) Eppendorf FemtoJet ${ }^{\oplus}$ microinjector

Fig. 2 Components of a typical microinjection system for injecting SCG neurons in dissociated cultures. The system shown does not include a $\mathrm{CO}_{2}$ supply 
motor module can be attached to the microscope via a manufacturer-specific attachment or can be positioned using a universal stand. Ideally, the microscope should have a heated stage. An environment chamber with regulated $\mathrm{CO}_{2}$ supply is optional.

2. FemtoTip ${ }^{\circledR}$ injection capillaries (Eppendorf) with screw-in thread for quick assembly into the capillary holder of the FemtoJet ${ }^{\circledR}$ microinjector (see Note 6).

3. $0.22 \mu \mathrm{m}$ centrifuge tube filter columns such as Corning ${ }^{\circledR}$ Costar $^{\circledR}$ Spin- $\mathrm{X}^{\circledR}$ centrifuge tube filters.

4. Eppendorf Microloader ${ }^{\mathrm{TM}}$ pipette tips for loading injection mix into injection capillaries and for removing any excess.

5. Sterile disposable blades for attachment to a nondisposable scalpel handle or sterile disposable surgical scalpels with a polystyrene handle. Blades should be curved with a relatively long cutting edge (e.g. No. 10 or No. 22).

\section{Methods}

\subsection{Dissociation and Culture of SCG Neurons}

All steps are performed at room temperature in a horizontal laminar flow cabinet, unless indicated otherwise.

1. Dissect superior cervical ganglia from mousepups at any age up to 2-3 days after birth as described in Chapter 2. Typically, $1-2$ ganglia will provide sufficient dissociated neurons for a single dish with a laminin-coated area of $7-10 \mathrm{~mm}$ in diameter. Nerve fibers, capillaries, or other encapsulating tissue should be cleaned from the ganglia before proceeding.

2. Transfer ganglia to $0.025 \%$ trypsin solution, prewarmed to $37{ }^{\circ} \mathrm{C}$, using a pair of sterile No. 5 forceps (sterilized in $70 \%$ ethanol and air dried before use). Use $5 \mathrm{ml}$ of trypsin solution in a $15-\mathrm{ml}$ tube for ganglia from multiple mice of the same genotype (up to 20-30) or, if processing individual mice separately, use $1 \mathrm{ml}$ of trypsin solution in separate $1.5 \mathrm{ml}$ tubes for each pair of ganglia. Incubate at $37^{\circ} \mathrm{C}$ in a waterbath for no more than 20-30 $\mathrm{min}$.

3. Transfer the ganglia to $0.2 \%$ collagenase solution, prewarmed to $37^{\circ} \mathrm{C}$, using a sterile $1 \mathrm{ml}$ tip attached to an appropriate pipetman/pipettor. Transfer in as small a volume of trypsin solution as possible. As in step 2 , use $5 \mathrm{ml}$ of collagenase solution in a $15-\mathrm{ml}$ tube for multiple ganglia or $1 \mathrm{ml}$ of collagenase solution in separate $1.5 \mathrm{ml}$ tubes for individual pairs of ganglia. Incubate at $37^{\circ} \mathrm{C}$ in a waterbath for no more than 20-30 min. 
4. Transfer the ganglia to a fresh $15 \mathrm{ml}$ tube or separate $1.5 \mathrm{ml}$ tubes, as appropriate, using a sterile $1 \mathrm{ml}$ tip attached to an appropriate pipetman/pipettor. Transfer in as small a volume of the collagenase solution as possible (ideally $<50 \mu \mathrm{l}$ ). Add $0.5 \mathrm{ml}$ of SCG medium and triturate the ganglia using a $1-\mathrm{ml}$ tip attached to an appropriate pipetman/pipettor by drawing the ganglia into the tip while it is pressed firmly to the base of the tube and then expel back into the tube normally. Repeat no more than ten times before transferring the cell suspension to a standard, uncoated $35-\mathrm{mm}$ tissue culture dish. If any ganglia remain intact, transfer them back into the tube in as small a volume of media as possible, and repeat the trituration step using another $0.5 \mathrm{ml}$ of SCG medium and add this to the previous cell suspension in the dish. Remove and discard any undissociated tissue remaining after the second trituration (see Note 7).

5. Preplating: Add additional SCG medium to the cell suspension in the standard, uncoated tissue culture dish to give a final volume of $1.5 \mathrm{ml}$, and incubate for $1-2 \mathrm{~h}$ in a $5 \% \mathrm{CO}_{2}$ incubator at $37^{\circ} \mathrm{C}$. Non-neuronal cells, appearing phase-dark on an inverted phase-contrast microscope, will adhere to the dish while the phase-bright neurons will remain in suspension. This step is not essential (see Note 8).

6. Transfer the cell suspension from steps $\mathbf{4}$ or $\mathbf{5}$ to a fresh $15-\mathrm{ml}$ tube or separate $1.5 \mathrm{ml}$ tubes, as required, using a $1-\mathrm{ml}$ tip attached to an appropriate pipetman/pipettor. Tap the dish several times if preplating was performed to maximize collection of neurons. Pellet cells at $200 \times g$ in a bench-top centrifuge for $5 \mathrm{~min}$.

7. Remove the majority of the supernatant leaving a small volume of medium $(<20-30 \mu \mathrm{l})$ at the bottom of the tube, so as not to disturb the cell pellet. Add sufficient fresh SCG medium for plating; 35-40 $\mu \mathrm{l}$ is sufficient for one dish with a laminincoated area 7-10 $\mathrm{mm}$ in diameter (see Note 9). Resuspend the cell pellet using a $1-\mathrm{ml}$ tip attached to an appropriate pipetman/pipettor by drawing the cell suspension into the tip while it is pressed to the base of the tube. Repeat five times with significantly less pressure than used during initial trituration (step 4).

8. Transfer 35-40 $\mu$ of neuronsuspension (or other appropriate volume as determined by cell counting, if performed) to the laminin-coated area of each poly-L-lysine/laminin-coated dish. The laminin solution should have been removed just prior to addition of the cell suspension. Avoid repeated pipetting that creates electrostatic forces that cause neurons to cluster in the 


\subsection{Microinjection \\ of SCG Neurons \\ and Neurite \\ Degeneration Assays}

center of the laminin-coated area. Incubate for $2 \mathrm{~h}$ in a $5 \% \mathrm{CO}_{2}$ incubator at $37^{\circ} \mathrm{C}$.

9. Check neurons have attached to the dish on an inverted phasecontrast microscope. Short neurites extending from the attached neurons should already be visible. Flood each dish with at least $1 \mathrm{ml}$ of SCG medium prewarmed to $37^{\circ} \mathrm{C}$.

10. Replace the SCG medium 1 day after initial plating and then every $2-3$ days thereafter. Neurons can be microinjected at any time from 3 days in vitro (DIV) onwards, although assays for injury-induced axon degeneration are best performed after 5 DIV when neurites are more developed.

1. Turn on the microinjector, micromanipulator, and microscope heated stage ( set to $37^{\circ} \mathrm{C}$, if present). The microinjector will take a few minutes to pressurize. If an environment chamber with $\mathrm{CO}_{2}$ supply is also present, set for a $5 \% \mathrm{CO}_{2}$ level and add some sterile, ultrapure water to a tray in the chamber to maintain humidity.

2. Prepare injection mix(es): These should be freshly prepared on the day of injection. For each mix, dilute water-dissolved test components (expression vectors, siRNAs, etc.) to $2 \times$ the desired final concentration in sterile, ultrapure water and combine with an equal volume of $1 \times \operatorname{PBS}(-)$ (withoutcalcium chloride and magnesium chloride). The final injection mix will thus have $\operatorname{PBS}(-)$ at $0.5 \times$ and each test component at the desired concentration. A $20-\mu \mathrm{l}$ mixture, combining $10 \mu \mathrm{l}$ of water-based components with $10 \mu \mathrm{l}$ of $1 \times \operatorname{PBS}(-)$, is usually sufficient for one injection session. An expression vector for a fluorescent protein or a fluorescently conjugated dextran (molecular weight of 10,000 or less) should be co-injected with the other components being tested, so that the injected neurons and their neurites can be visualized ( see Note 10). Stock solutions of conjugated dextrans are usually dissolved in $1 \times \operatorname{PBS}(-)$ and should thus be added as part of the $1 \times$ $\operatorname{PBS}(-)$ half of the injection mix. The total nucleic acid concentration in an injection mix should not exceed $200 \mathrm{ng} / \mu \mathrm{l}$.

3. Spin the injection mix through a $0.22-\mu \mathrm{m}$ centrifuge tube filter column in a microfuge at $14,000 \times g$ for $5 \mathrm{~min}$ to remove any particulate material that could block the injection capillary.

4. Load the injection mix into an injection capillary: Aspirate 2-3 $\mu$ l of injection mix into a microloader pipette tip using an appropriate pipetman/pipettor and expel directly into the tip end of the injection capillary while holding it vertically in its tip protector. Remove any air bubbles that might impede flow with gentle flicking or by aspirating with the microloader. Remove the tip protector and screw the loaded injection capillary into 
the FemtoJet ${ }^{\circledR}$ capillary holder, and attach the holder to the $\mathrm{x}, \mathrm{y}$, $\mathrm{z}$ motor module. Do not apply air pressure to the capillary until step 6.

5. Place a dish of dissociated SCG neurons into the dish holder on the microscope stage. Angle the injection capillary at $30-50^{\circ}$ from the horizontal, as preferred, and with the micromanipulator joystick set to coarse movements, position the tip of the injection capillary at the surface of the medium in the center of the microscope field of view. A combination of viewing from above and through the microscope using the low magnification objective (if present) will facilitate initial positioning. Once centered, set the micromanipulator joystick to fine movements, and viewing through the $32 \times / 40 \times$ objective, lower the capillary tip into the medium until it is positioned just above the SCG neurons on the base of the dish. The tip should be able to touch the surface of the SCG neurons within one-quarter turn of the joystick (which controls movement in the $z$ plane).

6. Microinject neurons in the dish: Set the compensation pressure (Pc) on the microinjector to $150-200 \mathrm{hPa}(1 \mathrm{hPa}=100$ Pascal $)$. The injection mix should flow out of the injection capillary continuously at a relatively slow rate (generally not visible). With the micromanipulator joystick still set to fine movements, position the injection capillary directly above the nucleus of an SCG neuron and lower until it enters the nucleus. If the injection mix is flowing correctly, the nucleus will swell. Allow the nucleus to expand by $\sim 10-20 \%$ (this should happen rapidly) and then remove the capillary tip by raising back to its starting position. Small lateral movements when lowering and raising the capillary tip will prevent hooking the neuron with the tip. A graphical representation of the injection procedure is shown in Fig. 3. Compensation pressure should be adjusted as needed to optimize the injection process; injection of too much mix at too fast a rate will kill neurons, whereas injection with too little will compromise visualization due to low fluorescent marker expression/concentration. Blockages in the tip that prevent flow even at high pressures can sometimes be resolved using the "clean" function on the microinjector (a short blast of high pressure to the capillary), but the capillary will need to be replaced if the blockage persists. While the number and location of neurons to be injected per dish will depend on the requirements of the assay being performed, neurite studies generally benefit from injection of relatively small numbers of neurons in relatively localized clusters/strips (see Note 11).

7. After injection, move the injection capillary back to its starting position and reduce the compensation pressure (Pc) on the microinjector to $0 \mathrm{hPa}$ before removing from the capillary holder. 


\section{Side view}
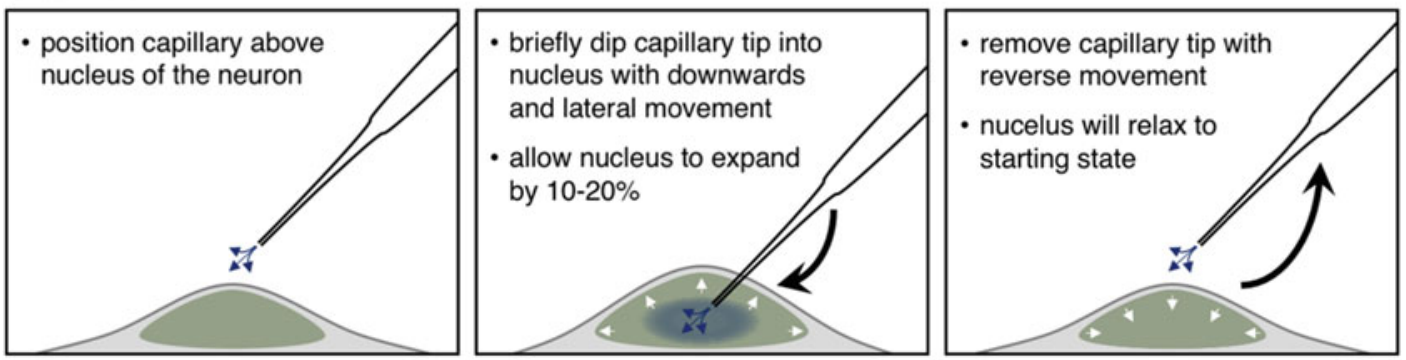

View from above (experimenter view through microscope eyepieces)
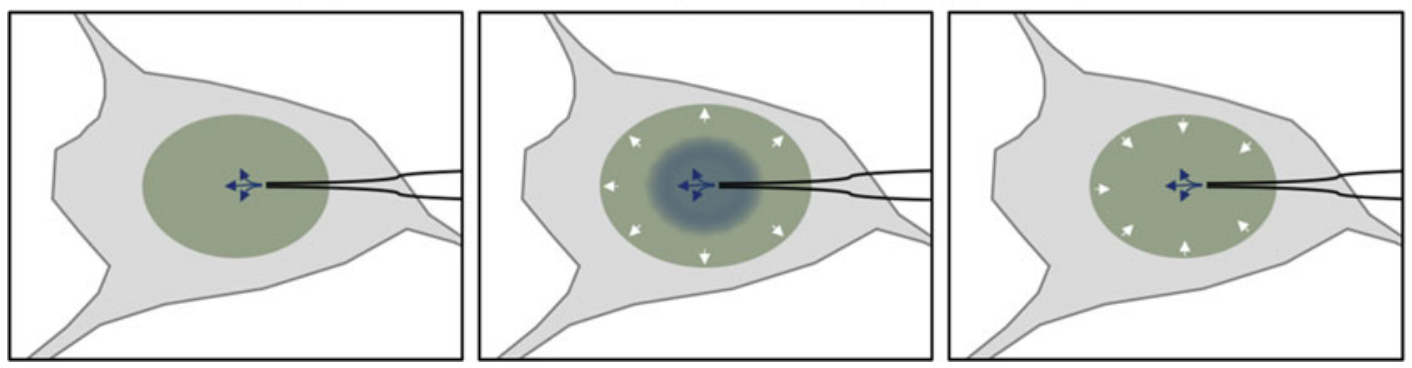

Fig. 3 Outline of the procedure for microinjecting dissociated SCG neurons. Graphical representations of a single neuron cell body from the side and from above are shown to illustrate key stages in the injection procedure. Written guidelines are provided at each stage. The view from above is a stylized view of what the experimenter will see in the center of the microscope field

8. Return the cultures to the $5 \% \mathrm{CO}_{2}$ incubator at $37^{\circ} \mathrm{C}$. It will take at least $24 \mathrm{~h}$ for the distal ends of long neurites of the injected neurons to become labeled, irrespective of the fluorescent injection marker used, but more proximal neurite regions will be visible immediately with conjugated dextrans and after just a few hours with an exogenously expressed fluorescent protein.

9. Optional transection of labeled neurites with a scalpel blade (see Note 12): After 24-48 h (to allow sufficient labeling of distal neurites), locate the cluster/strip of injected neurons in the dish on an inverted fluorescence microscope with the appropriate fluorescence filter and center the cell bodies in the field of view. Push the transmitted light arm of the microscope back to give access to the dish on the microscope stage. While viewing through a protective screen, use the circle of projected incident light in the dish as a guide to position the point of a curved, sterile surgical scalpel blade, so that a long, straight cut can be introduced to one side of the injected neuron cell bodies by rolling the cutting edge of the scalpel along the surface of the dish with a single down-and-up movement of the scalpel holder/handle (see Note 13). Avoid lateral movements of the blade that might unintentionally cause detachment of the 
neurite network by bringing the scalpel back to its tip before removing from the dish. If performed correctly, fluorescently labeled distal neurites should be completely separated from their cell bodies (Fig. 1).

10. Monitor/quantify neurite degeneration: Capture images of intact or transected fluorescently labeled neurites at various timepoints after initial injection or cutting (or both) on an inverted fluorescence microscope. Images captured using $10 \times$ or $20 \times$ objectives should provide sufficient resolution of individual neurites. What timepoints are used, and whether or not a cut is performed, will depend on the potential effect of the injected constructs and/or other reagents being tested. Healthy, intact neurites will retain a uniform fluorescent signal along their entire length, whereas the appearance of blebs or varicosities and/or a loss of continuity of the fluorescent signal are indicators of degeneration. For example, transected wildtype SCG neurites begin to show complete loss of continuity of the fluorescent signal from 6 to $8 \mathrm{~h}$ onward (Fig. 1). The number of intact neurites at any given timepoint can be compared to the number of intact neurites at a reference timepoint (usually $24 \mathrm{~h}$ after injection or just after cut) to calculate percentage survival/degeneration. The same field of neurites should be compared at each timepoint (see Note 14).

\section{Notes}

1. FBS is preferred over B27 supplement for dissociated SCG cultures and is essential when the neurons are to be microinjected. Sources of FBS should first be tested rigorously for their ability to maintain healthy cultures for at least 7-10 days. Different lots of FBS from the same source can also vary in their quality. Culture quality can be assessed by observing the morphology of the neuronal somas and neurites; somas should remain phase bright and have large, round nuclei with visible nucleoli while neurites should remain relatively smooth and free from blebs and develop into a dense network of neurite bundles.

2. Aphidicolin is used as an antimitotic to inhibit proliferation of non-neuronal cells. A combination of $20 \mu \mathrm{M}$ fluorodeoxyuridine and $20 \mu \mathrm{M}$ uridine can be used as an alternative to aphidicolin but is less potent.

3. 7S and 2.5S NGF can be obtained from a number of commercial sources. It is sensible to compare batches from different sources and to determine empirically the optimal concentration for healthy cultures. 
4. Low-sided dishes are used, so that as large an area as possible of the dish is accessible to the angled injection capillary when microinjecting. The coverslip insert base of ibidi dishes provides superior optical properties for imaging but is not essential. Laminin coating defines the area of the dish to which the neurons can attach and extend their neurites, and this can be varied, so that similar cell densities can be obtained with different numbers of neurons.

5. The methods described in this chapter are specific to the Eppendorf microinjection system detailed in Subheading 2.2. Use of other microinjection systems from other manufacturers may require a different injection technique, but the principles will be similar. Injections can be performed on a microscope without a heated stage and/or $\mathrm{CO}_{2}$ supply, but the time cultures are out of the $37{ }^{\circ} \mathrm{C}, 5 \% \mathrm{CO}_{2}$ incubator should then be limited to less than $15 \mathrm{~min}$ to avoid significant temperature/ $\mathrm{pH}$ stress.

6. Injection capillaries can alternatively be prepared from glass capillaries using a micropipette puller. A different, specialized capillary holder will be required for attachment of pulled capillaries to the FemtoJet ${ }^{\circledR}$ microinjector.

7. Trituration is the most critical step in the procedure. While insufficient trituration will result in retention of some neurons in clumps (not ideal for microinjection but still manageable), overtrituration can cause substantial loss of neuronal viability and should be avoided. The two phases of restricted trituration described in this method minimize the risk of overtrituration. If necessary, the state of dissociation at each stage can be monitored by looking at small aliquots of the cell suspension on an inverted phase-contrast microscope. The final cell suspension should contain mostly individual, phase-bright cells. Many will still have short neurite stumps attached.

8. The relatively small numbers of non-neuronal cells in dissociated mouse SCG neuron preparations can be managed adequately with antimitotics, such as aphidicolin, without performing the preplating step. In contrast, preplating is advisable if dissociating rat SCG neurons as non-neuronal cells are generally more abundant.

9. The yield of neurons per ganglion using this method is usually very reproducible, and the final cell density will generally be similar between preparations (assuming the same ratio of ganglia to plating area is used). However, if variable yield is found to be a problem, then a determination of cell concentration using a hemocytometer can ensure that equal numbers of neurons are plated with each preparation. 
10. The type of fluorescent marker used for visualization of the injected neurons and their neurites will depend on the potential outcomes of the assay being performed. Fluorescently conjugated dextrans will provide immediate labeling of injected neuron cell bodies and will rapidly diffuse into proximal neurites, but the signal will weaken as it diffuses along the neurites and becomes more diluted. In contrast, labeling will be relatively delayed using a fluorescent protein expression vector but will ultimately be brighter and more prolonged as everincreasing levels of the fluorescent protein spread into the neurites. Conjugated dextrans are thus more suited to situations where a manipulation induces rapid and early degeneration of otherwise uninjured neurites, while exogenously expressed fluorescent proteins are generally preferable in all other cases. However, it is important to consider possible toxic effects of exogenously expressed fluorescent proteins resulting from high or prolonged expression [7] and to be aware that the presence of a fluorescent protein expression vector can reduce expression from other co-injected constructs (and vice versa) as a result of competition between promoters for shared transcription factors. In general, it is best to use the lowest concentration of fluorescent protein expression vector that gives adequate visualization of neurites when these issues arise.

11. The dense neurite network in SCG neuron cultures largely consists of groups of neurites bundled together in small tracts rather than individual, isolated neurites. Microinjection of relatively small numbers of neurons thus increases the chances of visualizing and monitoring survival of individual neurites, even within a bundle, as opposed to the sum of the health of multiple neurites bundled together. The injection of neurons in relatively localized clusters or strips has advantages both in transection and nontransection assays: In transection assays, it ensures that the whole cluster/strip of injected cell bodies can easily be restricted to one side of the cut, so that the labeled neurites on the opposite side of the cut will all be transected (with a possible caveat-see Note 13), and in non-transection assays, it facilitates imaging of just the distal portions of the neurites, mostly in the same orientation, if an area distant to the cluster/strip of cell bodies is selected.

12. Individual neurites can be transected by laser ablation as an alternative to transection with a scalpel blade. Chemical insults can also be used to trigger neuritedegeneration that is mechanistically related to injury-induced (Wallerian) degeneration.

13. It is not necessary for the cut site to extend all the way across the cultured area of the dish when transecting neurites (indeed a complete bisection of the culture may increase the chance of 
detachment). However, because neurites in dissociated cultures are branched and sometimes follow a highly convoluted path, leaving a region of continuity of the neurite network around the ends of the cut site can result in untransected, fluorescently labeled neurites being present on the distal side of the cut. Any neurites being evaluated distal to the cut site that can be traced around the cut site should thus be excluded from the analysis.

14. Fluorescently labeled neurites undergoing degeneration initially develop variable numbers of neuritic swellings along their length, and this is followed by a loss of continuity. Swellings are very short lived when degeneration is rapid, for example, after injury, but can persist for longer before the loss of continuity following noninjury treatments that induce slower degeneration. Although the remnants of fully degenerated neurites can remain visible for several hours after degeneration, sometimes the fluorescent label fades/disintegrates to such an extent that the degenerated neurite effectively disappears. Calculations of the percentage of degenerated neurites (with swellings or discontinuity of the fluorescent label) in a single field at a single late timepoint can thus be an unreliable reflection of the actual extent of degeneration. A more accurate method is to follow the survival of exactly the same visual field of neurites and compare the number of healthy intact neurites (with continuous fluorescent labeling and no swellings) at each timepoint with that of the number present at an early, reference timepoint.

\section{Acknowledgments}

The authors were supported by funding from the UK Medical Research Council (grant number MR/N004582/1), Parkinson's UK (project grant G-1602), and a Sir Henry Wellcome postdoctoral fellowship from the Wellcome Trust (grant number 210904/ $\mathrm{Z} / 18 / \mathrm{Z})$.

\section{References}

1. Peng I, Binder LI, Black MM (1986) Biochemical and immunological analyses of cytoskeletal domains of neurons. J Cell Biol 102 (1):252-262

2. Conforti L, Gilley J, Coleman MP (2014) Wallerian degeneration: an emerging axon death pathway linking injury and disease. Nat Rev Neurosci 15(6):394-409. https://doi.org/ $10.1038 / \mathrm{nrn} 3680$
3. Gerdts J, Summers DW, Milbrandt J, DiAntonio A (2016) Axon self-destruction: new links among SARMl, MAPKs, and NAD+ metabolism. Neuron 89(3):449-460. https://doi. org/10.1016/j.neuron.2015.12.023

4. Whitfield J, Neame SJ, Ham J (2004) Methods for culturing primary sympathetic neurons and for determining neuronal viability. Methods Mol Biol 282:157-168. https://doi.org/10. 1385/1-59259-812-9:157 
5. Di Stefano M, Nascimento-Ferreira I, Orsomando G, Mori V, Gilley J, Brown R, Janeckova L, Vargas ME, Worrell LA, Loreto A, Tickle J, Patrick J, Webster JR, Marangoni M, Carpi FM, Pucciarelli S, Rossi F, Meng W, Sagasti A, Ribchester RR, Magni G, Coleman MP, Conforti L (2015) A rise in NAD precursor nicotinamide mononucleotide (NMN) after injury promotes axon degeneration. Cell Death Differ 22 (5):731-742. https://doi.org/10.1038/cdd. 2014.164

6. Gilley J, Adalbert R, Yu G, Coleman MP (2013) Rescue of peripheral and CNS axon defects in mice lacking NMNAT2. J Neurosci 33(33):13410-13424. https://doi.org/10. 1523/JNEUROSCI.1534-13.2013

7. Gilley J, Coleman MP (2010) Endogenous Nmnat2 is an essential survival factor for maintenance of healthy axons. PLoS Biol 8(1): el000300. https://doi.org/10.1371/journal. pbio. 1000300

8. Gilley J, Orsomando G, Nascimento-Ferreira I, Coleman MP (2015) Absence of SARMI rescues development and survival of NMNAT2deficient axons. Cell Rep 10(12):1974-1981. https://doi.org/10.1016/j.celrep.2015.02. 060

9. Loreto A, Di Stefano M, Gering M, Conforti L (2015) Wallerian degeneration is executed by an NMN-SARMI-dependent late $\mathrm{Ca}(2+)$ influx but only modestly influenced by mitochondria. Cell Rep 13(11):2539-2552 . https://doi.org/10.1016/j.celrep.2015.11. 032

10. Milde S, Coleman MP (2014) Identification of palmitoyltransferase and thioesterase enzymes that control the subcellular localization of axon survival factor nicotinamide mononucleotide adenylyltransferase 2 (NMNAT2). J Biol Chem 289(47):32858-32870. https://doi. org/10.1074/jbc.Ml14.582338

11. Milde S, Gilley J, Coleman MP (2013) Subcellular localization determines the stability and axon protective capacity of axon survival factor Nmnat2. PLoS Biol 11(4):e1001539. https:// doi.org/10.1371/journal.pbio.1001539

12. Hung CO, Coleman MP (2016) KIFlA mediates axonal transport of BACEl and identification of independently moving cargoes in living SCG neurons. Traffic 17(11):1155-1167. https://doi.org/10.1111/tra.12428

Open Access This chapter is licensed under the terms of the Creative Commons Attribution 4.0 International License (http://creativecommons.org/licenses/by/4.0/), which permits use, sharing, adaptation, distribution and reproduction in any medium or format, as long as you give appropriate credit to the original author(s) and the source, provide a link to the Creative Commons license and indicate if changes were made.

The images or other third party material in this chapter are included in the chapter's Creative Commons license, unless indicated otherwise in a credit line to the material. If material is not included in the chapter's Creative Commons license and your intended use is not permitted by statutory regulation or exceeds the permitted use, you will need to obtain permission directly from the copyright holder. 